\title{
Der Vater aller Dinge
}

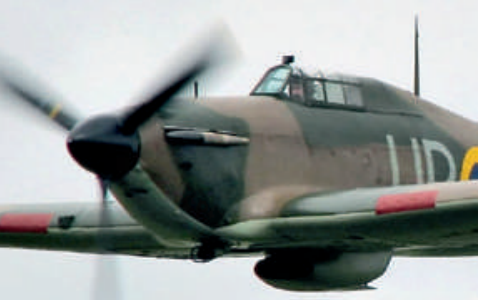

Überraschend: Zwischen Plexiglas-Cockpits alter Kampfflugzeuge und der Kataraktchirurgie gibt es einen Zusammenhang.
Beim Hantieren an einem Radargerät soll dem Elektroingenieur Percy Spencer ein Schokoladeriegel in der Hosentasche geschmolzen sein. Die sinkende Nachfrage nach dem Krieg zwang seine Firma, nach zivilen Anwendungsmöglichkeiten zu suchen. Es dauerte ziemlich lange, doch in den 1980er Jahren verhalfen die Singlehaushalte der Mikrowelle zum Durchbruch. Die Legende illustriert perfekt den Weg der modernen Technik: Aus der Rüstung kommt ein neues Produkt, das die Nachfrage zu einem Ding unseres Alltags macht. Dem Naturphilosophen Heraklit wird die mehrdeutige Sentenz «Der Krieg ist der Vater aller Dinge» zugeschrieben. Ohne Belagerungsgeräte keine neuen Baumaschinen, ohne Steigbügel aus Metall keine schwere Reiterei. Doch das Tempo dieser Innovationen hat seit der Industrialisierung rasant zugenommen.

Wie alle anderen Lebensbereiche ist auch die moderne Medizin von dieser väterlichen Herkunft geprägt. Denn nicht jede bahnbrechende Anwendung, wie zum Beispiel die Röntgenstrahlen, stammt aus der friedlichen Grundlagenforschung. Die grossen Kriege des vergangenen Jahrhunderts beschleunigten die Entwicklung neuer Technologien. Dem Sonarsystem zur Ortung von U-Booten entstammen die späteren Ultraschallgeräte, und aus der Chemie eines Kampfmittels wie Senfgas schufen Pharmakologen die Grundlagen der Krebstherapie. Die Zertrümmerung von Nierensteinen mittels Stosswellen verdankt ihren Ursprung dem Starfighter, einem Jagdflugzeug, dessen Metallverkleidung bei zweifacher Schallgeschwindigkeit durch Regentropfen beschädigt wurde. Bei der Analyse dieser Schäden durch die Dornierwerke entstanden unerwartet Geräte, die eine Verwendung in der Urologie und Orthopädie fanden. Berühmt ist die Geschichte der Intraokularlinsen. Am Anfang stehen die verletzten Bomberpiloten der Royal Air Force, denen die Splitter berstender Cockpits aus Plexiglas die Augen perforierten. Der Augenchirurg und spätere Sir Harold Ridley erkannte, dass dieses Material nicht mit dem Gewebe reagierte, und begründete 1949 die Kataraktchirurgie. Die Aufzählung liesse sich fast beliebig fortsetzen. Nicht immer ist die Herkunft derart eindeutig belegbar. Wenn T-Lymphozyten im schwerelosen Zustand, erzeugt durch den Parabelflug eines Tiger-Kampfjets, untersucht werden, ist das ein weiteres Beispiel, wie zivile und militärische Forschung ineinandergreifen. Ohne Elektronik keine Aviatik und schon gar keine Magnetresonanztomografie. Ohne Strahlenwaffen keine handlichen Laser-Skalpelle, ohne Militärrobotik keine Drohnen, aber auch einfachere Prothesen und weniger Technik im OP.
Gemäss Zahlen des Stockholmer International Peace Research Institute (SIPRI) arbeitet weltweit schätzungsweise die Hälfte aller wissenschaftlichen Forscher voll- oder teilzeitlich für militärische Projekte. Dazu gehören Grundlagenforschungen an Hochschulen oder Aufträge an private Unternehmen, die aus einem Verteidigungsbudget finanziert werden. Ohne Raketen und geheime Satelliten kein General-Positioning-System (GPS), kein bequemes Navi im Auto, keine präzise Meteo, kein effizienter Rettungsdienst. Was zivil hergestellt von militärischem Nutzen sein kann, unterliegt als Dual-useProdukt, das sind doppelverwendungsfähige Güter, einer Exportkontrolle. Für den umgekehrten Fall gibt es keine Ausfuhrlisten. Allenfalls spricht man von einem Spin-off-Ableger, der einen kleinen Teil der Rüstungskosten im zivilen Markt wieder einspielt. Auch Waffenverkäufe sind nie rentabel. Sie fördern die Geldverschwendung durch eine Rüstungsdynamik, deren Kosten selbst erfolgreiche Konsumprodukte nie decken werden. Schon Eisenhower, als USGeneral und späterer Präsident mit der Materie bestens vertraut, kritisierte in einer Rede den «militärisch-industriellen Komplex», von dem er sich als Politiker eingeengt fühlte.

Ist es völlig egal, woher unsere Gebrauchsgegenstände kommen? Der amerikanische Wissenschaftshistoriker Paul Forman (geboren 1937) formulierte in den 1970er Jahren eine These, wonach die militärische Finanzierung auf den Charakter und den Verlauf naturwissenschaftlicher Forschung einen grossen Einfluss hat. Heute bestreitet niemand, dass Wissenschaft nicht in einem wertfreien Raum vor sich geht. Wir sind eingebunden in einem militärisch-industriellen-wissenschaftlichen System, das längst alle Lebensbereiche, von der Radioastronomie bis zur Medizin, vom Cyberwurm Stuxnet bis zur Gesichtserkennung auf dem Handy, durchgestaltet. Dazu gehören auch die Biowissenschaften. Die zivilen Hersteller neuer Variationen des Vogelgrippevirus H5N1 wurden scharf kritisiert. Viele Forscher sind der Ansicht, dass diese Experimente hätten verhindert werden müssen, während die Befürworter den Nutzen für wirksame Impfstoffe gegen eine Pandemie hervorheben. Vermutlich haben beide recht. Die Studien wurden inzwischen publiziert. Es ist anzunehmen, dass in militärisch oder geheimdienstlich kontrollierten Labors ganz ähnliche Versuche am Laufen sind. Für Heraklit kam alles Seiende aus dem Feuer. 2500 Jahre und einige Atomkatastrophen später sind wir da nicht mehr so sicher.

Erhard Taverna 